Actes du 17e colloque du GERAS

\title{
LC/LCS : une langue paranoïaque?
}

\section{Nicole Décuré}

\section{OpenEdition}

\section{Journals}

Édition électronique

URL : http://journals.openedition.org/asp/3560

DOI : 10.4000/asp.3560

ISSN : 2108-6354

\section{Éditeur}

Groupe d'étude et de recherche en anglais de spécialité

\section{Édition imprimée}

Date de publication : 1 décembre 1996

Pagination : 273-279

ISSN : 1246-8185

\section{Référence électronique}

Nicole Décuré, «LC/LCS : une langue paranoïaque?», ASp [En ligne], 11-14| 1996, mis en ligne le 09 mai 2013, consulté le 03 mai 2019. URL : http://journals.openedition.org/asp/3560 ; DOI : 10.4000/ asp.3560

Ce document a été généré automatiquement le 3 mai 2019.

Tous droits réservés 


\title{
LC/LCS : une langue paranoïaque?
}

\author{
Nicole Décuré
}

Paranoïa

psychose chronique caractérisée par l'organisation

logique de thèmes délirants

comportement de quelqu'un, d'un groupe qui a tendance à se croire persécuté ou agressé (Le petit

Larousse illustré 1995)

1 Une branche de l'anglais de spécialité occupe une place à part car elle n'a pas à être enseignée. Au contraire, elle s'apprend et se manie en dehors du contexte scolaire. Il s'agit de la Langue de la Conspiration/Langue de Communication Sélective (LC/LCS) ou, en anglais(Foreign) Language for Conspiracy Purposes, soit (F)LCP. Examiner en quoi consiste cette langue nous a donné à réfléchir sur la notion même de communication, sur la motivation comme ressort principal d'apprentissage (d'une langue ou de tout autre objet) et sur l'utilisation des nouvelles technologies pour mener à bien une recherche.

Deux rencontres ont amené cette recherche : celle de Mona, une Égyptienne qui a appris le français en écoutant parler ses sœurs et pour comprendre ce qu'elles disaient; celle de Phil, un Américain qui, après un séjour en France, connaissait la langue mais ne la parlait pas ; vivre en France avec une Française n'avait pas suffi. De retour aux États-Unis, il s'est débloqué lorsqu'il a voulu communiquer à fins de conspiration pour faire des commérages avec sa femme sur les gens autour de lui sans être compris.

3 Partant de là, j'ai interrogé des enseignant(e)s de nombreux pays au cours d'un séminaire international à Salzburg. Parallèlement, j'ai posé la question suivante sur Internet aux membres de la liste de discussion TESL-L (13000 membres, en majorité enseignant l'anglais comme langue seconde). Dans ce type de situation, répond qui veut, qui se sent motivé(e).

13 April 1995

I have started a new research project on LCP i.e. language for conspiracy purposes. I mean by that a foreign language you use among a special group, family, friends, etc. so as not to be understood by the others. For example, Chinese schoolgirls may 
speak English among themselves so as not to be understood by the boys. I would appreciate any life story, experience that you can think of.

En soixante-douze heures, j'ai reçu seize réponses par courrier électronique et deux par courrier-escargot (dont une était une duplication du courrier électronique, par prudence) : dix le premier jour, cinq le deuxième jour, une le jour suivant.

J'ai formulé à nouveau ma demande en janvier 1996.

2 February 1996

In April, I asked TESL-L members for anecdotes about using a foreign language for conspiracy purposes, i.e. so as not to be understood. I got quite a few replies and have been able to start making categories but I was not on the net long enough to get enough stories. So I need a few more. Has anybody got any more stories to tell? Thanks.

5 Dans les trois jours, j'ai reçu douze réponses de plus, y compris celle d'une femme qui avait déjà répondu la première fois longuement et, de toute évidence, ne s'en souvenait pas. Les réponses ont été étalées sur six jours, à raison de deux ou trois par jour. Tous les messages ont été analysés, les divers éléments classés. Beaucoup de réponses comprennent plusieurs éléments.

\section{La langue, outil de communication}

6 Une langue étrangère est considérée comme un outil pour communiquer le plus largement possible avec le maximum de gens les plus divers. L'arrivée rapide, successivement, des télex, fax, Minitel, téléphone portable (avec les conversations privées devenues publiques sur les quais de gare), tous les services du téléphone (avis d'appel, conférences téléphoniques, etc.), la télévision par satellite, Internet, tout ceci trahit une aspiration à une communication rapide, efficace, large, un désir de communiquer tout de suite et n'importe quand, et d'être accessible aux autres en tout lieu et tout instant.

7 Les humains, avec une certaine partialité, considèrent les humains comme les êtres les plus évolués dans l'ordre animal à cause de cette capacité langagière, orale et a fortiori écrite. Le mot communication lui-même est devenu un mot clé, pour ne pas dire passepartout, en toutes circonstances. Tout le monde communique tout le temps et en ceci réside l'espoir, pour les optimistes, que la communication mène à la compréhension et pourquoi pas à la paix. Il y a une sorte de croyance angélique dans ce pouvoir de la communication bien que, et ceci devrait nous mettre la puce à l'oreille, les moyens de communication aient toujours joué un rôle crucial dans les guerres et autres formes de violence, en commençant par le cheval de Troie qui n'était après tout qu'une autre forme de moyen de communication. Les Indiens savaient ce qu'ils faisaient quand ils coupaient les fils du télégraphe (si l'on en croit les westerns).

8 Le télégraphe devint extrêmement populaire en Angleterre en 1845 quand il permit à la police d'arrêter un meurtrier (Le livre mondial des inventions 1991 : 85). Toujours à en croire le cinéma, la première action d'un cambrioleur, d'un kidnappeur, d'un violeur ou d'un meurtrier est d'arracher les fils du téléphone lorsqu'il pénètre dans une maison, empêchant ainsi la communication, alors perçue comme l'espoir, voire la vie. La marine et les boy-scouts ont au moins un point en commun : on y enseigne encore le morse. On sait aussi que les développements qui se sont fait jour dans les méthodes d'apprentissage des langues pendant et après la Seconde guerre mondiale, à l'époque de la guerre froide, sont dus aux besoins de l'armée américaine et de la C.I.A. réunies. Une des participantes à 
l'enquête décrite ici mentionne les besoins du F.B.I. en connaissance du thaï, du russe ou du vietnamien pour lutter contre les criminels étrangers sur le sol américain.

9 Car on oublie qu'une langue étrangère sert aussi à ériger une barrière entre soi et les autres, que c'est un moyen de couper la communication, que ce soit parmi les criminels ou la population honnête.

10 Pourquoi ? Comment? Quelles implications cela a-t-il pour l'enseignement/apprentissage des langues?

11 Au-delà de la discussion sur LC/LCS, somme toute anecdotique, sera abordée la façon dont cette recherche a été menée: par le biais d'Internet comme source de données. On évoquera quelques pistes possibles pour l'avenir de la recherche avec cet outil.

\section{La conspiration}

12 D'après Robert Wernick (1994: 109), dans la Genèse, la conspiration est la première activité humaine, avant le sexe. Il y a conspiration lorsque deux individus ou plus s'entendent pour commettre un acte illégal ou poursuivre un but légal par des moyens illégaux.

13 Il n'y a rien d'illégal dans la conspiration telle que je l'entends, mais elle donne le frisson du hors norme, hors société, sans qu'on encoure de risque. Elle satisfait ce besoin, semble$\mathrm{t}$-il, profond, de clan: nous contre les autres. Les scouts, les enfants, les adolescents aiment avoir un langage secret compris d'eux seuls ce qui fait de la LCS une «langue paranoïaque ». Le langage est à la « safe conspiracy » ce que la capote est au « safe sex ».

Le tableau suivant résume les différences entre la « vraie » conspiration et l'emploi d'une LCS.

Tableau 1

\begin{tabular}{|l|l|}
\hline \multicolumn{1}{|c|}{ Conspiration } & \multicolumn{1}{|c|}{ LCS } \\
\hline Illégale & Rarement \\
\hline Trahison possible des sous-fifres, des acolytes & Solidarité, cohésion \\
\hline $\begin{array}{l}\text { Besoin d'avouer, de se vanter } \\
\text { Un bon conspirateur se tait }\end{array}$ & $\begin{array}{l}\text { Conspiration au grand jour } \\
\text { Un bon conspirateur parle }\end{array}$ \\
\hline $\begin{array}{l}\text { Mythe populaire: } \\
\text { Les conspirations sont puissantes. }\end{array}$ & Prendre son destin en main \\
\hline $\begin{array}{l}\text { Mythe populaire: } \\
\text { Exotisme des conspirations }\end{array}$ & Beaucoup de langages « exotiques » \\
\hline $\begin{array}{l}\text { Conspiration (« thriller } \text { ) }=\text { inscription pathologique } \\
\text { dans un monde ordonné }\end{array}$ & $\begin{array}{l}\text { Introduction d'un ordre privé par } \\
\text { rapport à un monde hostile ou } \\
\text { incohérent ou refusé }\end{array}$ \\
\hline La conspiration rend le monde opaque aux autres. & id. \\
\hline
\end{tabular}




\begin{tabular}{|l|l|}
\hline $\begin{array}{l}\text { Conspirateurs : pas toujours des étrangers, mais au } \\
\text { moins des « marginaux » }\end{array}$ & «L'ennemi » est « étranger », autre. \\
\hline $\begin{array}{l}\text { Histoires d'espions : questions de langage (langue } \\
\text { étrangère, langue codée, chiffrée, encre invisible, } \\
\text { microfilms, etc.) }\end{array}$ & Langage au centre de la conspiration \\
\hline
\end{tabular}

La majorité des répondants sur Internet sont des Américains (2/3). On pourrait en conclure hâtivement que les Américains complotent/conspirent plus que les autres et cela pourrait illustrer l'affirmation de Davis selon laquelle les Américains conceptualisent leur relation au reste du monde en termes de conspiration contre eux, trait caractéristique d'une nation qui cherche son identité (Brandt 1982 : 63). Les Américains voient des conspirations partout, même s'ils se sont dotés du plus formidable système de conspiration avec la C.I.A. Mais le public de TESL-L est fortement américain, donc cette conclusion est à écarter (pour l'instant).

Internet lui-même repose sur une contradiction : créé à des fins de conspiration, il sert de moyen de communication de masse tous azimuts (une personne peut s'adresser à une autre en privé ou à des milliers, voire des millions en public) et, accessoirement, par une mise en abîme, il sert aussi de moyen d'explorer les différentes façons de conspirer.

17 Le code privé permet de communiquer à tout petit nombre par rapport au langage ambiant que tout le monde comprend. Sur une liste de discussion, on peut parler à tout le monde ou s'adresser à quelqu'un en particulier. Tout ce qu'il faut, c'est le bon code d'accès qui se résume à une formule.

\section{Analyse des réponses : le pourquoi et le comment de la conspiration}

18 On peut placer les réponses dans deux grandes catégories fonctionnant l'une sur l'exclusion et l'autre sur le clan. Les deux approches reviennent au même; seul le point focal est différent.

19 1. Communiquer en cercle fermé, sans être compris(es) des autres et/ou exclure les autres apparaît fréquemment dans les réponses (39). Les différents cadres évoqués sont ceux de la guerre, de la guerre froide, du crime organisé, de la lutte pour le pouvoir. Sur le plan de la vie privée, nombre de réponses mentionnent la conspiration contre ses propres enfants, notamment avec l'utilisation du yiddish, tradition qui semble se perpétuer avec les générations et qui motive les enfants pour apprendre. Les chiens sont aussi concernés par la conspiration en langue "étrangère ». Sont également mentionnés le désir de maintenir une vie privée par rapport à l'environnement, de se différencier des autres, de s'identifier à un groupe minoritaire, de critiquer sans être comprise, discuter un prix à payer, tricher, s'amuser aux dépens des autres, discuter leurs problèmes, garder un secret, exclure d'autres groupes, régler des conflits, préparer une bonne surprise, etc.

20 2. Le clan fonctionne aussi par l'exclusion, de façon plus positive. Il s'agit, avant tout, de créer un cercle fermé, une intimité, une complicité, un cocon au sein de la famille, contre une autre culture, contre un autre clan (10 réponses). La priorité est au bien-être au sein du clan. 
21 3. Refuser de parler une langue que l'on connaît (5 réponses) revient au même que parler une langue que les autres ne comprennent pas et peut servir à des buts aussi divers que s'isoler de ses compatriotes à l'étranger, éviter les conflits ou même, par une stratégie alambiquée, obliger quelqu'un à parler, obtenir des renseignements : en parlant une langue inconnue d'abord puis une langue connue des interlocuteurs/trices, le soulagement éprouvé par ces dernier(e)s les amène à être plus serviables.

4. Mais la conspiration peut échouer (3 réponses), par exemple par un effet boomerang quand on se croit, à tort, non compris. Les enfants, à force d'écouter les parents parler une langue inconnue, arrivent à l'apprendre. Cet échec est positif et débouche sur le point qui nous intéresse.

\section{Implications pour l'enseignement/l'apprentissage des langues}

Il est prouvé, une fois de plus, que la motivation personnelle, la force des circonstances sont des moteurs principaux, pour ne pas dire primordiaux. Dans le cas de la LCS, il n'y a aucun souci de «correction ». L'essentiel est d'être compris(e) par son interlocuteur/ trice.

Mais comment canaliser cette motivation vers la classe ? Les langues modimes ${ }^{1}$, plus que l'anglais, pourraient trouver là un ressort puissant.

\section{Implications pour la recherche}

Les caractéristiques des répondant(e)s mettent à jour autant de questions que de réponses et permettent de définir des champs de recherche ultérieurs, notamment sur l'utilisation d'Internet comme outil pour la recherche.

Par exemple, la prépondérance des Américains en TESL-L (2/3) pose problème car les réponses manquent de diversité. D’autres pays ont une approche différente du plurilinguisme: l'Afrique du Sud, la Suisse, nombre de pays d'Afrique où les langues coexistent. De même, les réponses sont faites aux deux tiers par des femmes. Est-ce parce que les femmes sont majoritaires dans l'enseignement des langues ou parce qu'elles conspirent plus? Quelle proportion de femmes y a-t-il dans le réseau ? Ce pourcentage vient contredire l'idée reçue que $10 \%$ seulement de femmes se servent d'Internet. Les enseignantes sont-elles une race à part ?

\section{Perspectives}

Cette recherche précise, si elle doit être poursuivie, devrait s'élargir à d'autres publics et tenter de répondre aux questions laissées en suspens, notamment sur l'utilisation de la motivation de parler une LCS dans l'apprentissage des langues.

Surtout, cette recherche démontre un phénomène qui se développe sur Internet : on peut maintenant interviewer un grand nombre de gens très divers, tâche impossible auparavant par manque de moyens humains et financiers. Il n'y a pas d'accumulation de papiers, on ne garde que l'essentiel et les données entrent d'elles-mêmes dans l'ordinateur. Les réponses arrivent très rapidement ou pas du tout. 

les carences du réseau TESL-L montrent l'impérieuse nécessité de la formation d'un réseau de ce type français, ou francophone, peut-être européen, plus centré sur nos préoccupations. C'est à quoi nous allons nous employer.

\section{BIBLIOGRAPHIE}

Brandt, B. E. 1982. « Reflections of 'the paranoid style' in the current suspense novel ». Clues: A

Journal of Detection 3/1, 62-69.

Davis, D. B. (dir.). 1971. The Fear of Conspiracy: Images of un-American Subversion from the Revolution to the Present. Ithaca : Cornell University Press.

Fulcher, J. 1983. « American conspiracy: Formula in popular fiction ». The Midwest Quarterly 24/2, 152-164.

Le livre mondial des inventions. 1991. Paris : Compagnie 12/Fixot.

Palmer, J. 1978. Thrillers: Genesis and Structure of a Popular Genre. Londres : Edward Arnold.

Wernick, R. 1994. « Don't look now - But all those plotters might be hiding under your bed». Smithsonian 24/3, 108-124.

\section{NOTES}

1. Langues modimes = langues moins diffusées-moins enseignées : néologisme dû à Charles Barrière, responsable du programme Lingua à Bruxelles.

\section{RÉSUMÉS}

Une langue étrangère est considérée comme un outil pour communiquer le plus largement possible avec le maximum de gens les plus divers. Mais on oublie qu'une langue étrangère sert aussi à ériger une barrière entre soi et les autres, que c'est un moyen de couper la communication. Cette recherche est le résultat d'une enquête menée auprès de personnes qui ont employé et emploient une langue étrangère afin de n'être comprises que d'un ou quelques individus. Ceci nous amène à nous interroger sur la notion de communication, sur la motivation pour apprendre une langue étrangère et sur Internet comme nouvel outil de recherche.

A foreign language is considered as a tool to communicate as widely as possible with the largest number of people on various subjects. But it is also used to cut off communication, to raise a barrier between people. This research was undertaken to probe into the reasons why, how and with whom people use a foreign language to communicate selectively with a limited number of 
people. It has led to a reflection about the notion of communication, on motivations to learn a foreign language and on the use of Internet as a new research tool.

\section{INDEX}

Mots-clés : communication sélective, conspiration, motivation, recherche

Keywords : conspiracy, research, selective communication

\section{AUTEUR}

\section{NICOLE DÉCURÉ}

Nicole Décuré est maître de conférences à l'UFR de Langues vivantes et responsable du laboratoire LAIRDIL à l'Université Paul-Sabatier Toulouse 3. Ses publications récentes en didactique des langues sont : «Jouer? Est-ce bien raisonnable ?, Les Langues modernes 2, 1994, 16-24 ; « Un test, ça se teste », Cahiers de LAIRDIL 1, 1994, 43-54 ; « The difficulties of teaching a 'man-made language' », Women and Language 17/1, 1994, 36-37 ; « Soixante-quinze minutes en ballon : étude de l'utilisation d'un module de gestion sur vidéodisque pour la pratique de l'anglais en autonomie ", Cahiers de l'APLIUT 14/2, 1994, 20-31; « To see or not to see », Cahiers de LAIRDIL 3, 1994, 27-31; « Poèmes et jeu de quilles : introduction et bibliographie », Les Langues modernes 4, 1995, 7-12 ; « La parole sans douleur », Cahiers de LAIRDIL 4, 1995, 45-58 ; « L'arbre qui cache la forêt : incidence de la lecture préalable d'un texte français sur la compréhension d'un texte anglais », version courte : Actes du Colloque sur la lecture à Toulouse 1, 1995 ; version longue : Cahiers de LAIRDIL 6. decure@cict.fr 